\title{
LA APLICACIÓN DEL MÉTODO DE ISÓTOPOS ESTABLES A MÁRMOLES EXPLOTADOS EN ÉPOCA ROMANA EN LA MITAD SUR DE LA PENÍNSULA IBÉRICA
}

\author{
POR \\ AURELI ÁLVAREZ PÉREZ \\ Departament de Cristal.lografia i Mineralogia, Universitat Autònoma de Barcelona \\ MARC MAYER OLIVÉ \\ LITTERA, Universitat de Barcelona \\ ISABEL RODÁ DE LLANZA \\ LEMLA, Universitat Autònoma de Barcelona
}

\section{RESUMEN}

Se pretende hacer un estudio de los resultados del método de isótopos estables como medio discriminatorio para los mármoles de la mitad sur de la Península Ibérica (especialmente Macael y Almadén de la Plata), así como también una aproximación arqueológica a su estudio, identificación y difusión.

\section{SUMMARY}

Isotopic readings are employed to distinguish marbles from the southern-half of the Iberian Peninsula, and in particular those from Macael and Almadén de la Plata. The study, identification, and distribution of these marbles are given an archaeological perspective.

\section{ASPECTOS PETROLÓGICOS ${ }^{1}$}

El análisis del mármol a través de las técnicas propias de la petrología, en especial de la observación sobre lámina delgada mediante el microscopio petrográfico, permite detectar tanto los componentes mineralógicos como su estructura y sus características genéticas. Aunque esta información es muy valiosa para la identificación de los distintos tipos de mármoles, esta técnica, como otras, no deja de tener sus limitaciones y sus dificultades. Por ello, los diversos autores siempre han preconizado el empleo simultáneo de diversas técnicas para validar los datos obtenidos ${ }^{2}$.

En este sentido, el análisis químico elemental ha sido una de las técnicas más empleadas hasta el

\footnotetext{
1 Esta parte ha sido redactada por Aureli Álvarez.

2 L. Moens-P. de Paepe- M. Waelkens, «Multidisciplinary Research and Cooperation: Keys to the Successful Provenance Determination of White Marble», Ancient Stones: Quarying, Trade and Provenance, Acta Archaeologica Lovaviensia 4, Lovaina 1992, p. 247; L. Moens-P. Roos-P. de
}

punto de que en algunos momentos ha sido aplicada de modo casi exclusivo, obviando de una manera inexplicable el estudio petrográfico. Los resultados obtenidos, no siempre suficientemente satisfactorios, han llevado a la utilización de otras técnicas complementarias entre las que cabe destacar como más reciente el estudio de los isótopos estables ${ }^{18} \mathrm{O}$ y ${ }^{13} \mathrm{C}$ presentes en los componentes carbonatados. Últimamente este tipo de estudios ha proliferado tanto que la información acumulada ha propiciado el intento de producir una base de datos que pueda servir cada vez más para caracterizar los distintos lugares de producción conocidos ${ }^{3}$.

A fin de intentar establecer las posibilidades reales de este método para la identificación de áreas fuente, presentamos una serie de análisis efectuados sobre muestras de mármoles utilizados en época romana procedentes de la mitad sur de la Península Ibérica. Tanto las canteras como las muestras han sido perfectamente documentadas y los análisis han sido efectuados por los Servicios Científico-Técnicos de la Universidad de Barcelona.

A modo de consideración general sobre los mármoles, indicaremos que son rocas producidas por el metamorfismo que ha actuado sobre sedimentos car-

Paepe-R. Lunsingh Scheurleer, «Provenance Determination of White Marble Sculptures from the Allard Pierson Museum in Amsterdam, Based on Chemical, Microscopic and Isotopic Criteria», ibidem, p. 269. Un útil cuadro de las diferentes técnicas puede verse en M. Mariottini, «La provenienza dei marmi cristallini usati in antico: un problema aperto», Marmi antichi II, ed. P. Pensabene = Studi Miscellanei 31 (1998), pp. 23-34, esp. 25-27 (cuadro p. 27) y para la aportación de los isótopos, pp. 29-33.

${ }^{3}$ L. Stowell Pearson-N. Herz, «Isotopic Analysis of a Group of Roman Gorgon Sarcopghagi», Ancient Marbles..., cit., p. 293; K. Ramseyer et alii, «Provenance Investigation of Marble Artifacts now in the Collection of the Museum of Art and History in Geneva», ibidem, p. 287. 
bonatados a los cuales ha transformado, originando una intensa recristalización y eventualmente la aparición de nuevos minerales. El tipo y la composición de la roca carbonatada y el tipo de metamorfismo que ha actuado son, evidentemente, los principales condicionantes del fraccionamiento isotópico que ahora detectamos. Pero nadie ignora que otras causas secundarias han podido influir en este fraccionamiento y es, precisamente, este segundo aspecto el que proporciona mayores argumentos para aplicar este método a la determinación de áreas fuente. En efecto, el conjunto global de circunstancias puede ser tal que solamente se dé en un único yacimiento y puede, de este modo, ser utilizado para identificarlo.

Según J.R. O’Neil ${ }^{4}$ la variación en el fraccionamiento de los isótopos ${ }^{18} \mathrm{O}$ y ${ }^{13} \mathrm{C}$ depende de los procesos físicos y químicos que tienen lugar en la Naturaleza, de modo que los valores actuales medibles en una muestra vienen determinados por fenómenos cinéticos y están fijados por las leyes de la termodinámica. Para elementos pesados intervienen, además, reacciones nucleares.

Las moléculas que contienen el isótopo pesado son más estables y tienen una energía de disociación más elevada que las que contienen el isótopo más ligero. Los principales factores que influyen en el fraccionamiento isotópico son la temperatura, la composición química, la estructura cristalina y la presión. Los enlaces con un elevado potencial iónico y baja masa atómica tienden a incorporar preferentemente isótopos pesados. Por su parte, los carbonatos son ricos en ${ }^{18} \mathrm{O}$ porque el oxígeno está unido a un carbono que es un átomo pequeño y tiene una elevada carga iónica.

Si bien gran parte de los mármoles están compuestos mayoritariamente por carbonato cálcico, algunos tienen porcentajes variables y significativos de dolomita (carbonato cálcico-magnésico) que pueden influir en el valor de fraccionamiento isotópi$\operatorname{co}^{5}$. J.R. O'Neil ${ }^{6}$ ya había constatado una mayor tendencia a la concentración de isótopos densos de la dolomita, a causa de la presencia de la molécula de $\mathrm{CO}_{3} \mathrm{Mg}$. Así fijó para el $\mathrm{Mg}$ un coeficiente de 31.2 frente al 28.0 de la calcita y el 28.7 del aragonito. El fraccionamiento de ${ }^{18} \mathrm{O}$ a $25^{\circ} \mathrm{C}$ en el ara-

${ }^{4}$ J.R. O'Neil, «Theoretical and Experimental Aspects of Isotopic Fractionation», Reviews in Mineralogy 16 (Stable Isotopes) (1986), pp. 1-40.

5 S.I. Golyshev-N.L. Padalko-S.A. Pechenkin, «Fractionation of Stable Oxygen and Carbon Isotopes in Carbonate Systems», Geochemistry International 10 (1981), pp. 85-99.

6 J.R. O’Neil-R.N. Clayton-T.K. Mayeda, «Oxygen Isotope Fractionation in Divalent Metal Carbonates», Journal of Chemical Physics 51 (1969), pp. 5547-5558. gonito y en la calcita difiere muy poco ya que es del orden de 0.5-0.6\% ${ }^{7}$.

Según S.I. Golyshev et alii ${ }^{8}$, el fraccionamiento isotópico en carbonatos viene determinado principalmente por el radio catiónico, y la masa empieza a ser importante a partir de valores altos, como el bromo $(\mathrm{Br})$ y el plomo $(\mathrm{Pb})$. Por su parte, la estructura cristalina es menos importante que el enlace y que la misma composición del mineral. Finalmente, la presión tiene un efecto prácticamente despreciable, dado que apenas hay cambio de volumen y que la abundancia de ${ }^{18} \mathrm{O}$ en los minerales es solamente del 0.2 por mil.

Una vez hechas estas consideraciones generales, queremos precisar que el presente trabajo no tiene una finalidad exhaustiva sino que se pretende más bien una exposición que sirva para establecer las posibilidades y las limitaciones del análisis isotópico como medio para determinar la procedencia de los mármoles arqueológicos. En este sentido, se ha procedido a la selección de muestras de canteras bien definidas y documentadas, excepto en el caso de las procedentes de la Sierra de Mijas que han sido incluidas con la finalidad de tener información de los materiales de esta formación geológica, muy particular, que proporcionó materiales marmóreos abundantes (Coín, Mijas...). Estas últimas muestras han sido seleccionadas con criterios exclusivamente geológicos, dado que, aunque hubo explotación romana en la zona, no han sido recogidas en cantera.

Del conjunto muestreado se ha realizado el estudio petrológico mediante lámina delgada. Sólo a título orientativo cabe indicar que los mármoles procedentes de Mijas son esencialmente dolomíticos y que los mármoles de Cabezo Gordo son «cipollinos» con abundancia de micas y clorita. Los mármoles restantes pueden llegar a ser muy parecidos entre sí y, en ocasiones, difíciles de diferenciar; son de tamaño de grano grueso, con cristales bien conformados y contactos netos que presentan una estructura granular muy bien desarrollada. Algunos mármoles de Almadén de la Plata presentan deformaciones postmetamórficas.

El número de muestras no es excesivo y consideramos que es el mínimo que permite obtener conclusiones orientativas. Hemos preferido una mayor diversidad de canteras (siete en total) por encima de una densificación de muestras.

Los resultados analíticos, fruto de los datos ob-

${ }^{7}$ M. Robinson-R.N. Clynton, «Carbon-13 Fractionation between Aragonite and Calcite», Geochemica Cosmochemica Acta 33 (1969), pp. 997-1002.

8 Cf. nota 5. 
tenidos en el laboratorio, han sido agrupados en la Tabla 1 donde puede verse, además, el conjunto de muestras pertenecientes a cada uno de los diversos yacimientos.

TABLA 1

\begin{tabular}{|c|c|c|c|c|}
\hline Yacimiento & $N^{\circ}$ muestra & $\begin{array}{c}\delta^{18} \mathrm{O} \text { (PDB) } \\
\pm 0.3 \% \\
\end{array}$ & $\begin{array}{c}\delta^{{ }^{13}} \mathrm{C} \text { (PDB) } \\
\pm 0.1 \% o \\
\end{array}$ & $\mathrm{~N}^{\circ}$ laboratorio \\
\hline \multirow[t]{6}{*}{ Almadén de la Plata } & 5413 & -5.99 & 2.84 & JA202 \\
\hline & 5415 & -5.30 & 3.29 & JA201 \\
\hline & 5416 & -5.24 & 2.81 & JA203 \\
\hline & 5417 & -5.92 & 2.82 & JA206 \\
\hline & 5418 & -6.11 & 3.25 & JA204 \\
\hline & 5419 & -5.63 & 2.96 & JA205 \\
\hline \multirow[t]{7}{*}{ Cabezo Gordo } & 5858 & -6.11 & 2.75 & JA12 \\
\hline & 5860 & -6.16 & 2.81 & JA26 \\
\hline & 5863 & -5.81 & 2.75 & JA10 \\
\hline & 5864 & -4.25 & 2.62 & JA11 \\
\hline & 5866 & -4.24 & 2.54 & JA7 \\
\hline & 5868 & -4.97 & 2.55 & JA8 \\
\hline & 5870 & -4.87 & 2.35 & JA9 \\
\hline \multirow{5}{*}{ Estremoz } & 5150 & -5.54 & 2.01 & JA6 \\
\hline & 5154 & -5.96 & 2.14 & JA41 \\
\hline & 5155 & -5.59 & 2.03 & JA37 \\
\hline & 5159 & -5.94 & 1.36 & JA33 \\
\hline & 5162 & -6.18 & 1.13 & JA5 \\
\hline \multirow[t]{3}{*}{ Macael blanco } & 8065 & -1.83 & 2.24 & JA39 \\
\hline & 8099 & -2.81 & 2.61 & JA36 \\
\hline & 8100 & -3.27 & 2.66 & JA40 \\
\hline \multirow{5}{*}{ Macael gris } & 8093 & -5.71 & 2.74 & JA38 \\
\hline & 8094 & -3.47 & 2.48 & JA34 \\
\hline & 8095 & -2.71 & 3.01 & JA42 \\
\hline & 8096 & -1.80 & 2.84 & JA35 \\
\hline & 8097 & -5.05 & 2.20 & JA31 \\
\hline \multirow[t]{5}{*}{ Borba } & 5991 & -6.20 & 1.39 & JA13 \\
\hline & 5992 & -7.31 & 0.85 & JA1 \\
\hline & 5993 & -6.20 & 0.75 & JA4 \\
\hline & 5994 & -6.57 & 1.09 & JA3 \\
\hline & 5996 & -5.83 & 0.89 & JA2 \\
\hline \multirow[t]{7}{*}{ Mijas } & 5673 & -3.76 & 3.12 & JA17 \\
\hline & 5674 & -2.51 & 3.19 & JA16 \\
\hline & 5678 & -14.15 & 0.02 & JA14 \\
\hline & 5681 & -1.23 & 4.33 & JA18 \\
\hline & 5683 & -3.55 & 3.11 & JA15 \\
\hline & 5684 & -2.20 & 3.25 & JA19 \\
\hline & 5685 & -0.83 & 3.38 & JA20 \\
\hline
\end{tabular}

Estos mismos resultados han sido expuestos en una gráfica cuyos ejes son, respectivamente, las concentraciones de ${ }^{18} \mathrm{O}$ (abcisas) y de ${ }^{13} \mathrm{C}$ (ordenadas).

Como es normal, existen en el gráfico zonas de clara superposición y zonas bien separadas, lo cual condicionará evidentemente la posibilidad de utilizar esta información con fines discriminatorios. En líneas generales, las canteras relacionadas con el anticlinal de Estremoz (Portugal) aparecen agrupadas indicando la homogeneidad de los mármoles en él desarrollados, fruto de unas condiciones sedimentarias bastante semejantes y producto de un mismo metamorfismo. En su conjunto, estas canteras se diferencian (ver línea divisoria) del resto de canteras estudiadas, pertenecientes a la parte oriental de la Península. Entre estas canteras, las muestras procedentes de Mijas quedan separadas del resto; ello puede explicarse porque se trata de mármoles dolomíticos y tienen una fragmentación isotópica distinta a la de los mármoles calcáreos. No está clara la división entre los productos de las canteras de Macael (variedad blanca y variedad gris) de las variedades procedentes de Cabezo Gordo (Murcia) y éstas, a su vez, tampoco están bien separadas de las muestras procedentes de Almadén de la Plata. Sin embargo, estas últimas están bien separadas de las muestras de Macael.

Los materiales de Cabezo Gordo, al tratarse de mármoles «cipollinos», presentan una variabilidad importante y muestran caracteres propios que coinciden con los de otras canteras, más definidas en cuanto a sus propiedades. Almadén de la Plata tiene muestras parecidas a las de Cabezo Gordo por cuanto las canteras se hallan en el núcleo de un anticli- 




que se prepara mediante una homogeneización y un cuarteo a partir siempre de una cantidad suficiente de material que sea representativa según la variabilidad de la piedra (mármol en este caso). El sentido que tiene en este caso una muestra de tan sólo $10 \mathrm{mg}$ es que con ello evitamos estropear la pieza, al tomar tan pequeña cantidad. Pero una muestra tomada de esta manera puede no ser representativa, puesto que todas las muestras que se vayan obteniendo en estas condiciones darán, probablemente, resultados distintos; baste ver la dispersión de datos en el diagrama de la figura 2.

Por tanto, la ventaja que con nal que reproduce condiciones metamórficas semejantes a las de Cabezo Gordo. En su conjunto son diferentes a las de Macael.

Como se ve, la Geología ayuda a explicar la variabilidad de resultados.

L. Moens et alii ${ }^{9}$ son muy explícitos en el sentido de reconocer que, a la vista de los resultados del análisis isotópico: «....seuls quelques marbres méditerranéens peuvent être identifiés à l'aide de leur signature isotopique». Evidentemente el gráfico no deja lugar a dudas (figura 2).

En el extremo opuesto parece situarse N. Herz ${ }^{10}$ al afirmar que: «Stable isotopic signatures are the most widely used system today for determining marble provenance». Añade que una de las ventajas del análisis isotópico es la pequeña cantidad de muestra, $10 \mathrm{mg}$, que se necesita. Sin embargo, este extremo es discutible; efectivamente, cuando en análisis se requiere una pequeña cantidad de muestra, ésta no se toma directamente sobre la piedra sino

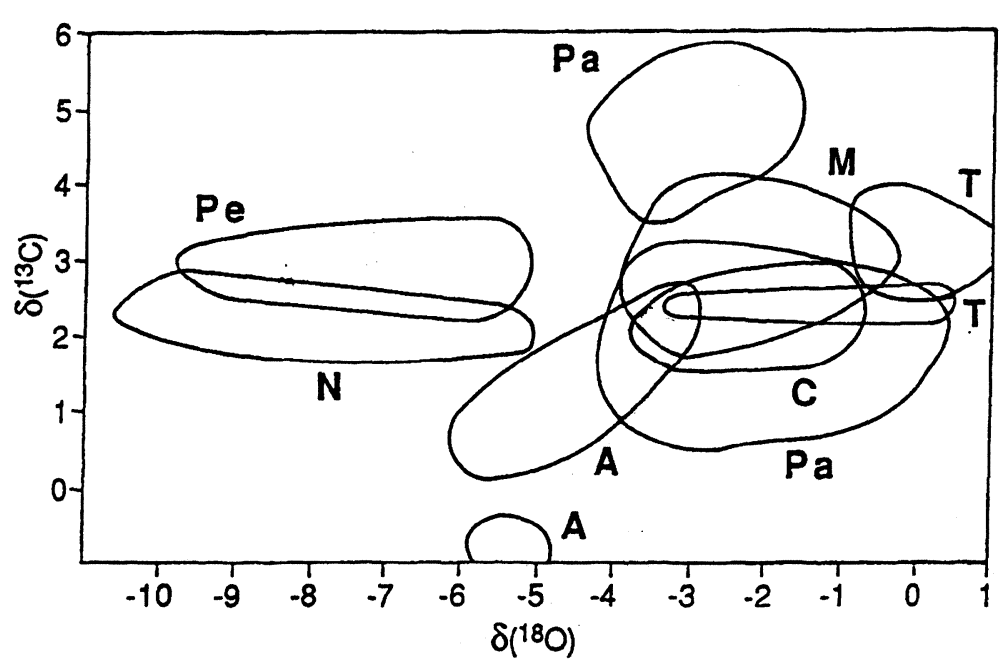

Figura 2.

${ }^{9}$ L. Moens-P. de Paepe-M. Walkens, «Une approche multidisciplinaire permettant de determiner l'origine des marbres blancs utilisés pendant l'Antiquité classique», Les marbres blancs des Pyrénées. Approches scientifiques et historiques. Entretiens d'Archéologie et d'Histoire 2, Saint-Bertrand-deComminges 1995, pp. 33-47.

${ }^{10} \mathrm{~N}$. Herz, «Stable Isotope Applications to Problems of Classical Greek and Roman Marbles: Provenance Authenticity and Assembly of Artifacts», Les marbres blancs des Pyrénées... cit., pp. 11-23.

diagrama de la figura 2, el mármol del Pentélico y el de Carrara caen en dominios separados; podría, por lo tanto, aplicarse el análisis isotópico.

Otro aspecto a tener en cuenta es el número total limitado de puntos que pueden situarse dentro del dominio de los intervalos de ${ }^{18} \mathrm{O}$ y ${ }^{13} \mathrm{C}$ que pueden presentar los mármoles. Este será, a su vez, el núme- 




ro máximo de análisis diferentes que podremos encontrar. Consecuencia directa es que, por fuerza, muchos análisis coincidirán entre sí, a pesar de ser de canteras distintas. Cuantos más yacimientos queramos distinguir, más tupida será la red de puntos. ¿Cuál es el límite máximo de yacimientos o canteras que pueden ser consideradas en un diagrama ${ }^{18} \mathrm{O} /$ ${ }^{13} \mathrm{C}$ ? ¿Cuál es el número máximo de muestras que se pueden dibujar en el diagrama? ¿Cuál es la diferencia mínima entre los valores obtenidos para dos muestras para decir que son realmente distintas?

Una posible respuesta a estas preguntas queda plasmada en la figura 3, donde se han superpuesto los datos obtenidos en este trabajo a los datos ofrecidos por L. Moens et alii ${ }^{11}$.

La figura 3 nos permite observar cómo los datos procedentes de Almadén de la Plata caen dentro del campo determinado por los datos procedentes del mármol pentélico. Así pues, dos mármoles de origen y características petrográficas distintos, presentan una misma composición isotópica. La mayoría de las muestras de Mijas se asemeja al mármol de Mármara (Proconeso). Las muestras de Cabezo Gordo, en nada semejantes al mismo, aparecen en el campo del mármol pentélico. El Macael blanco es más disperso y sus muestras se asemejan tanto al mármol de Carrara (con el cual es inconfundible desde el punto de vista petrográfico) como al mármol de Tasos o de Mármara, éstos, a su vez, englobados por el mármol de Paros. Como se ve, la confusión está servida. Únicamente un pequeño grupo de mármoles de Estremoz y de Borba quedan fuera de los dominios señalados en la figura 2. Estos mármoles habían sido ya estudiados por J.M.P.

\footnotetext{
11 Cf. nota 9.
}

Cabral et alii desde el punto de vista del método isotópico ${ }^{12}$.

Responder adecuadamente a todas las preguntas planteadas no es tarea fácil. Se está en el inicio del método que, por otra parte, ya ofrece, como hemos visto, limitaciones muy concretas a la hora de interpretar procedencias ${ }^{13}$. Por mucho que este método, junto a otras técnicas analíticas modernas, pueda considerarse prometedor por parte de algunos autores, es difícil creer que se llegue al deseo expresado por L. Moens et alii ${ }^{14}$ : «On peut espérer que les données fournies par ces techniques seront suffisamment discriminantes pour pouvoir remplacer l'information apportée par la pétrographie et l'analyse chimique». En este caso, por ahora poco probable, el mármol se convertiría en un acervo de datos numéricos susceptibles de ser tabulados por el analista, que perdería de vista toda connotación con la realidad petrográfica y las características geomorfológicas.

\section{ASPECTOS ARQUEOLÓGICOS ${ }^{15}$}

Las consideracions petrológicas dan unos resultados que conviene contextualizar desde un punto de vista arqueológico. No se trata en este caso del estudio de las canteras, bien identificadas, sino de la dispersión de los materiales estudiados y de su correcta valoración en el panorama lapídeo que presenta la Hispania romana como componente indispensable de su paisaje arquitectónico, decorativo, escultórico y epigráfico.

En época reciente un artículo de $\mathrm{S}$. Walker y $\mathrm{K}$. Matthews ${ }^{16}$ se planteaba la necesidad del «ojo práctico», es decir de la observación macroscópica para

12 J.M.P. Cabral et alii, «Preliminary Study on the Isotopic and Chemical Characterization of Marbles from Alto Alentejo (Portugal)», Ancient Marbles..., cit., pp. 191 ss.

${ }_{13}$ K. J. Matthews-L. Moens-S. Walker-M. Waelkens-P. de Paepe, «The Re-evaluation of Stable Isotope Data for Pentelic Marble», Ancient Stones..., cit., pp. 203 ss.

14 Cf. nota 9.

15 Esta parte del trabajo ha sido redactada por Marc Mayer e Isabel Rodá, pertenecientes respectivamente al Laboratori d'Investigació i Tractament de Textos i Epigrafia Romans i Antics (LITTERA), i al Laboratori per a l'Estudi dels Materials Lapidis de l'Antiguitat (LEMLA).

16 «Stable Isotope Analysis of Carrara Marble: Some Questions for the Archaeologist», Marble. Art Historical and Scientific Perspectives on Ancient Sculpture (Malibu 1988), Malibó 1990, pp. 125-134. 
distinguir algunos de los mármoles cuyas «signatures», aunque muy claras y específicas, se superponían. Así resultaba muy claro para la primera autora que el arqueólogo debe replantearse con su experiencia, incluso visual, algunos de los resultados analíticos, en especial cuando proceden de distintas fuentes o, si se quiere, trabajos analíticos. Lo mismo sucede cuando, a pesar de los análisis, se opina sobre la antigüedad o incluso refacciones de piezas y elementos estudiados.

En esta línea, en la que primeramente se han demostrado los resultados y las limitaciones de los análisis isotópicos aplicados a los mármoles del sur de la Península Ibérica, nos vamos a mover en las páginas que siguen donde intentaremos contrastar arqueológicamente los resultados obtenidos al tiempo que intentaremos una contextualización territorial de los mismos, teniendo en cuenta, además, las aportaciones anteriores.

Los estudios sobre los mármoles hispanos han sido objeto de interés sólo en época relativamente reciente puesto que hace poco más de dos decenios que aparecieron los trabajos pioneros de A. Tavares, A.M. Canto y W. Grünhagen ${ }^{17}$.

El de A. Tavares, vinculado a las excavaciones lusofrancesas de Conimbriga, presentaba una primera aproximación a los materiales lapídeos explotados en época romana en Portugal, con resultados analíticos ${ }^{18}$. A.M. Canto, por su parte, trataba en su trabajo del conjunto de las canteras hispano-romanas, o explotadas posiblemente por los romanos ${ }^{19}$. El trabajo de W. Grünhagen versaba sobre Munigua e intentaba identificar el conjunto de materiales presentes en el yacimiento, proponiendo variedades hispanas y utilizando fundamentalmente datos obtenidos a partir del uso comercial actual de los mismos ${ }^{20}$; sus resultados, aunque la experiencia los ha desmentido en buena parte, abrieron también una brecha aprovechada para la realización de estudios posteriores.

17 A. Tavares, «Matériaux de construction et de décoration», J. Alarcão-R. Etienne, Fouilles de Conimbriga I. L'architecture, París 1977, pp. 271-277; A.M. Canto, «Avances sobre la explotación del mármol en la España romana», Archivo Español de Arqueología 135-138 (1977-1978), pp. 165-188; W. Grünhagen, «Farbiger Marmor aus Munigua», Madrider Mitteilungen 19 (1978), pp. 290-306.

18 A Tavares, «Matériaux de construction...», cit., pp. 271-277 donde publica un análisis petrológico de los materiales presentes en Conimbriga.

19 A.M. Canto, «Avances...», cit., el mapa de ubicación de las canteras.

${ }^{20}$ Las láminas de color en el trabajo de W. Grünhagen permiten contrastar las identificaciones realizadas mediante la ayuda de un marmolista comercial para aproximar las variedades a la producción española actual; no obstante, no escapaba al autor la posibilidad de que se tratara de variedades importadas. Movidos por las láminas de color, realizamos una inspección ocular de los materiales de Munigua en
Es sintomático que estos estudios, de la década de los setenta ${ }^{21}$, abordaran la analítica petrológica aplicada a los materiales arqueológicos, el inventario de los recursos lapídeos de Hispania y una técnica macroscópica de identificación de materiales, orientada fundamentalmente a identificar las variedades locales peninsulares.

La década de los 80 supuso un fuerte empuje para estos estudios ya que vieron la luz trabajos analíticos, petrológicos y arqueológicos abundantes, así como algunos intentos de síntesis ${ }^{22}$. En la actualidad, algunas de las variedades hispanas forman parte ya de los repertorios internacionales ${ }^{23} \mathrm{y}$, a nivel local, disponemos ya de estudios fiables tanto de variedades en yacimientos arqueológicos como de canteras ${ }^{24}$. Ha llegado, en consecuencia, el momento de enfrentarse al problema más espinoso de cuantos se plantean al identificar

una visita al yacimiento en el año 1992 con acceso a los materiales de los almacenes; pudimos comprobar entonces la presencia de un abundante número de materiales importados, según recogemos en M. Mayer-I. Rodá, «The Use of Marble and Decorative Stone in Roman Baetica», The Roman Archaeology Conference (Reading 1995), en prensa. Debemos agradecer a los Profs. Th. Hauschild y F. Chaves el haber podido consultar estos materiales.

${ }^{21} \mathrm{Cf}$., además de la bibliografía citada en n. 17, R. Gnoli, Marmora romana, Roma 1971 (2. ed. Roma 1988) y la nueva aproximación en G. Borghini ed., Marmi antichi, Roma 1989, pp. 13-42.

22 M. Cisneros, Mármoles hispanos: su empleo en la España romana, Zaragoza 1988; M.P. Lapuente-M. Cisneros-M. Ortiga, «Contribución a la identificación de mármoles españoles empleados en la Antigüedad», Noticiario Arqueológico Hispánico 30 (1988), pp. 257-274; M. Mayer, «Aproximación al problema de la importación del mármol en la Hispania romana», PACT 27. Le commerce maritime des romains (Barcelona 1988), Rixensart 1990 (pub. 1995), pp. 265-277, esp. pp. 269 y 271 para los mármoles que aquí nos ocupan.

23 F. Braemer, «L'ornementation des établisements ruraux en Gaule et dans les régions limitrophes», Actes du Colloque La Villa romaine (Paris 1981), Caesarodunum 21 (1982), pp. 53-74; Id., «Le commerce des matériaux d'architecture et de sculpture de part et d'autre de la chaine des Pyrénées dans les provinces de Tarraconaise, de Narbonaise et d'Aquitaine», 106 Congrès National des Sociétés Savantes, Perpiñán 1981, pp. 52-72; Id., «Répertoire des gisements de pierres ayant exporté leur production à l'époque romaine», Les ressources minérales et l'histoire de leur exploitation, París 1986 , pp. 287-328. Cf. bibliografía citada en la nota siguiente.

${ }^{24}$ A. Álvarez-M. Mayer, «Materiales lapídeos de origen local utilizados en la costa sur del litoral catalán», VI Congreso Español de Estudios Clásicos, vol. II, Sevilla 1981, pp. 303310; M. D. del Amo, «Aportación al estudio de las canteras romanas de la zona arqueológica de «Els Munts», Estudis Altafullencs 5 (1981), pp. 5-25; F. Braemer, «L'ornamentation...», cit.; M. Martín-Bueno-M. Cisneros, «Aproximación al estudio de materiales de construcción romanos de Bílbilis (Calatayud, Zaragoza)», XVII Congreso Nacional de Arqueología (Logroño 1983), Zaragoza 1985, pp. 875-879; A. M. Mayer-A. Álvarez-I. Rodá, «La importación del mármol en época romana. El ejemplo de Ventimiglia y su contraposición con el litoral norte de la Tarraconense, Quaderni di Studi 
las variedades presentes en los yacimientos y su procedencia: se trata, ni más ni menos, que de establecer criterios discriminantes entre las variedades marmóreas blancas y grises ${ }^{25}$.

Es un hecho sabido que este problema de discriminación y clasificación ha preocupado a quienes se

Lunensi 10-12 (1985-1987), pp. 497-523; S.F. Ramallo-R. Arana, Canteras romanas de Carthago Nova y alrededores (Hispania Citerior), Murcia 1987; M. Recasens, «Tarraco y el comercio del mármol en época romana a través del estudio de sus capiteles», Pyrenae 21 (1987), pp. 123-128; M.P. Lapuente-M. Cisneros-A. Ortiga, «Contribución a la identificación...», cit.; M. Cisneros, «Sobre la explotación de calizas en el sur de España en época romana: canteras de Gador (Almería), Atarfe (Granada), Antequera (Málaga) y Cabra (Córdoba), Caesaraugusta 66-67 (1989-1990), pp. 128-132; A. Álvarez, «La procedència dels materials lapidis», en Ted'a, Un abocador del segle $V$ d.C. en el fòrum provincial de Tarraco Tarragona 1989, pp. 395-402; M.L. Loza-J. Beltrán, La explotación del mármol blanco de la Sierra de Mijas en época romana. Estudio de los materiales arquitectónicos, escultóricos y epigráficos, Faventia Monografíes 10, Bellaterra 1990; M. Mayer-I. Rodá, «El comercio del mármol en el Mediterráneo y su reflejo en la ciudad romana de Sagunt», Saguntum y el mar, Valencia 1991, pp. 37-45; M. Mayer, «L'explotation des ressources lapidaires en Hispania», Les Dossiers d'Archéologie 173 (1992), pp. 16-20; L. Segura, «Explotación romana de las canteras de 'mármol rojo' de Cabra», Actas del I Coloquio de Historia Antigua de Andalucía (Córdoba 1988), ed. J. F. Rodríguez Neila, vol. II, Córdoba 1993, pp. 111-124; I. Rodá, «Los materiales de construcción en Hispania», XIV Congreso Internacional de Arqueología Clásica (Tarragona 1993), Tarragona 1994, vol. 1, pp. 323-334, esp. pp. 327-331; M. Mayer, «La circulación de marmor Numidicum en Hispania», L'Africa romana. Atti dell'XI Convegno di Studio (Cartago 1994), Ozieri 1996, vol. II, pp. 837-848; I. Rodá, «Los mármoles de Itálica. Su comercio y origen», Italica como excepción. Jornadas Académicas del MMCC aniversario de la fundación de Italica (Sevilla 1994), Sevilla 1997, pp. 155-180; M. Mayer-I. Rodá, «The Use of Marble...», cit.; I. Rodá, «I marmi romani di Hispania: estrazione e lavoro», Harmony in Stone: Shaping, Building and Decoration Techniques through History (Pula 1996), vol. Pre-Actas, Materijali br. 8, Pula 1996, pp. 21-22 y 69-70; M. Mayer, «Sobre las calizas amarillas de la franja costera de la Hispania Citerior», Marmi antichi II, cit., pp. 99-110; R. Falcone-L. Lazzarini, «Note storico-scientifiche sul broccatello di Spagna», ibidem, pp. 87 97; A. Álvarez-M. Mayer, «Aproximació als materials lapidis decoratius presents al jaciment de Can Modolell (Cabrera de Mar, Maresme). Estudi volumètric i comparatiu», De les estructures indígenes a l'organització provincial romana de la Hispània Citerior, edd. M. Mayer-J.M. Nolla-J. Pardo, «ÍTACA. Annexos» 1, Barcelona 1998, pp. 43-49; I. Rodá, «La explotación de las canteras en Hispania», Hispania. El legado de Roma. Zaragoza 1998, pp. 113-118; M. Mayer-I. Rodá, «El broccatello de Tortosa: testimonios arqueológicos», Hommage à Claude Domergue (en prensa); A. Álvarez-M. MayerI. Rodá, El marmor de Tarraco (en prensa).

${ }^{25} \mathrm{Cf}$. para el caso de los mármoles griegos los trabajos pioneros de N. Hertz-W. Kendrick Pritchett, «Marble in Attic Epigraphy», American Journal of Archaeology 57,2 (1953), pp. 71-83; C. Renfrew-J.S. Peacey, «Aegean Marble: a Petrological Study», Annual of the British School at Athens 63 (1968), pp. 45-66. Cf. también el volumen recopilatorio de J.B. Ward-Perkins, Marble in Antiquity, edd. H. Dogel-B. Ward-Perkins, Archaeological Monographs of the British School at Rome 6, Londres 1992. han dedicado al tema desde el siglo XIX ${ }^{26}$. Se ha aplicado una amplia gama de métodos analíticos a este objetivo con resultados tan sólo parcialmente satisfactorios. Desde la década de los setenta se inició la técnica de análisis basada en la identificación de isótopos estables que ha resultado singularmente fiable como factor discriminatorio, en especial si se combina con otras técnicas petrológicas en el caso de los mármoles blancos ${ }^{27}$. Los datos técnicos podrán hallarse pormenorizados en los apartados correspondientes a los análisis que presentamos en la primera parte de este trabajo.

Las variedades hispanas de mármol blanco o grisáceo no son pocas, pero en este caso y para este primer ensayo de aproximación a este método de análisis, nos hemos limitado a unas variedades concretas de procedencia bien establecida, con evidencias concretas de utilización en época romana y a partir de muestras de cantera.

Algunas de estas variedades han sido objeto ya de importantes estudios arqueológicos e incluso analíticos y otras lo serán seguramente en el futuro, dada la importancia de las explotaciones y la extensión del uso de sus materiales en época romana. La selección que presentamos abarca, recordemos, siete canteras que representan un total de 38 muestras.

Las canteras son en primer lugar las de Borba y Estremoz, que corresponden al anticlinal de Estremoz en el Alto Alentejo (Portugal). De estas dos procedencias se había realizado ya un análisis mineralógico, químico e isotópico por parte de un equipo portugués ${ }^{28}$ cuyos resultados contrastamos con los aquí obtenidos, aunque el estudio al que nos referimos abarcaba un mayor número de canteras de dicho anticlinal ${ }^{29}$.

El uso de los materiales marmóreos de esta procedencia es un hecho bien probado e incluso han sido identificados restos de la explotación romana en canteras de Vila Viçosa y Rio de Moinhos ${ }^{30}$. La dispersión de sus materiales es importante en toda

26 G.R. Lepsius, Griechische Marmorstudien, Berlín 1890; M. Moltesen-N. Herz-J. Moon, «The Lepsius marbles», Ancient Stones..., cit., pp. 277-281.

${ }^{27} \mathrm{Cf}$. la bibliografía citada en la nota 9 .

28 J.P.M. Cabral et alii, «Preliminary Study...», cit., pp. 191-198; cf. la bibliografía anterior, especialmente los trabajos de F. Gonçalves, recogida en p. 194.

29 J.M.P. Cabral et alii, "Preliminary Study...», cit., pp. 191-198 estudian un total de 28 muestras procedentes de 10 canteras y localizaciones distintas; cf. esp. cuadro I y fig. 1 .

30 J. Alarcão-A. Tavares, «A Roman Marble Quarry in Portugal», Studia Pompeiana and Classica in Honor of Wilhelmina F. Jashemski, 2. Classica, ed. R.I. Curtis, New Rochelle, N.Y. 1989, pp. 1-12; J.M.P. Cabral et alii, «Preliminary Study...», cit., pp. 191-192; J. D'Encarnação, Inscrições romanas do Conventus Pacensis, 2 vols., Coimbra 1984, passim, cf. esp., vol. 2, pp. 821-822. 
la Lusitania romana, con una abundante presencia en su capital Augusta Emerita (Mérida) desde los retratos más antiguos ${ }^{31}$ y con notabilísimos ejemplos de material arquitectónico decorativo y escultórico, bien patentes, por ejemplo, en el llamado foro de mármol ${ }^{32}$. P. Pensabene ha propuesto por su parte la existencia de officinae en las tres capitales provinciales hispanas que se habrían formado en Roma o en otras ciudades itálicas, trabajando arquitectura oficial y traspasarían a Hispania los modelos de la capital utilizando mármoles tanto locales (Mérida) como importados (Tarragona) ${ }^{33}$.

El mármol de Borba-Estremoz parece haber llegado a Italica (Santiponce, Sevilla) y quizás a Baelo (Bolonia, Cádiz), donde entra en competencia con el mármol de Almadén de la Plata ${ }^{34}$.

La presencia de los mármoles alentejanos en yacimientos portugueses es muy frecuente y su irradiación se expande por Andalucía oriental, donde abunda, por ejemplo, en piezas de revestimiento y arquitectónicas de Munigua (Mulva, Sevilla) ${ }^{35}$.

El mármol que macroscópicamente más se asemeja a ciertas variedades de los anteriores es la variedad blanca y la ligeramente rosada de las canteras de Almadén de la Plata, aunque, como hemos visto, se agrupan de manera diferenciada según el análisis isotópico.

La calidad del mármol blanco de Almadén de la Plata, de grano grueso y transparente, puede llegar

31 Para los antiguos retratos emeritenses, T. Nogales, El retrato privado en Augusta Emerita, Badajoz 1997, esp. núms 3-15, pp. 6-23; Id., en La mirada de Roma, TarragonaMérida-Toulouse 1995, pp. 213-214.

32 J.M. Álvarez Martínez-T. Nogales Basarrate, «Schema urbain de Augusta Emerita: le portique du forum», Akten des XIII. Internationalen Kongresses für klassische Archäologie (Berlín 1988) Berlín 1990, pp. 336-338; W. Trillmich, «Los tres foros de Augusta Emerita y el caso de Corduba», Colonia Patricia Corduba. Una reflexión arqueológica (1993), ed. P. León, Córdoba 1996, pp. 175-188; Id., «Reflejos del programa estatuario del forum Augustum de Mérida», II Reunión sobre Escultura romana en Hispania (Tarragona 1995), Tarragona 1996, pp. 95-108.

${ }_{33}$ P. Pensabene, «Costruzioni pubbliche e committenza nella Spagna romana», Homenatge a F. Giunta. Committenza e committenti tra Antichità e Alto Medioevo (Erice 1994), ed. M. Mayer-M. Miró, Cornucopia 3, Barcelona 1996, pp. 123-182, esp. 158-159.

${ }^{34}$ Cf. para Itálica, I. Rodá, «Los mármoles de Itálica...», cit., pp. 161 y 174-176; M. Mayer-I. Rodá, «The Use of Marble...», cit. En Baelo, según observación macroscópica, pudo ser empleado el mármol de Estremoz en dos altares, uno con decoración relivaria conservado todavía en el templete situado al oeste de la fuente semicircular del foro que cierra la terraza que da acceso al Capitolio, y otro inscrito, decorado asimismo con objetos rituales similares (cf. J.-N. Bonneville-S. Dardaine-P. Le Roux, Belo V. L'épigraphie. Les inscriptions romaines de Baelo Claudia, Madrid 1988, núm 14, pp. 37-38).

${ }_{35}$ Cf. la bibliografía citada en las notas 17 y 20. a confundirse macroscópicamente con variedades de mármol de Paros muy apreciadas. En este lugar pueden observarse restos evidentes de la explotación antigua en tal extensión que merecerían un tratamiento monográfico; los loci de extracción parecen todavía bien definidos y organizados ${ }^{36}$. La difusión epigráfica de este mármol como soporte de textos es muy importante, como lo era también la de las variedades alentejanas ${ }^{37}$. Su presencia en los yacimientos béticos es muy elevada; así podemos constatar su gran abundancia en Italica (donde constituye, además, un material decorativo esencial del Traianeum) ${ }^{38}$, Singili Barba (cortijo de El Castillón, Antequera, Málaga), Córdoba, en el teatro de Malaca (Málaga), en Urso (Osuna), Munigua, en el teatro de Gades (Cádiz) ${ }^{39}$. Está presente también en Mérida, como hemos dicho, y en Castulo (Cazlona) ${ }^{40}$.

Una vía que queda por investigar es si realmente la irradiación de este mármol cruza el estrecho de Gibraltar y se expande por el norte de África, lo cual es muy probable dadas las corrientes comerciales entre la Bética y el litoral marroquí; pensemos, además, en la ausencia de canteras de mármol en esta zona norteafricana y en la posibilidad de que las de Almadén de la Plata (antiguo Pagus Marmorarius) pudieran haber sido de propiedad imperial. En este sentido una primera investigación en Lixus y Tingis podría resultar sintomática y quizás reveladora.

El tercer material que presenta en sus variedades blancas un gran parecido con los del Alentejo y de

${ }^{36}$ A.M. Canto, según sus informaciones verbales y en «Avances...», cit., pp. 176-177, y en Arqueología 79, Madrid 1980 , p. 202, realizó unas primeras prospecciones y un trabajo de excavación en la zona de Los Covachos, con resultados positivos respecto a las trazas de explotación antigua. En tres sucesivas visitas (1981, 1994 y 1996) hemos detectado restos de explotaciones tradicionales de muy diversa cronología y, en la parte superior de esta área, hemos detectado la existencia de pequeños loci, bien cortados con paredes de separación que parecen corresponder a explotaciones antiguas, quizá romanas.

${ }^{37} \mathrm{Cf}$. para el conventus Pacensis, nota 30. El mármol de Almadén de la Plata constituye un material abundantemente empleado como soporte de las sencillas y numerosas placas funerarias béticas (cf. G. Fabre-M. Mayer-I. Rodá, «Inscripciones 'alienae' en Museos y colecciones de la provincia de Barcelona», Ampurias 44 (1982), pp. 213, 217-218, 234235). Respecto a la utilización epigráfica de los mármoles de Estremoz, cf. las identificaciones de M. Cisneros, Mármoles hispanos..., cit., p. 159 para Regina.

${ }^{38}$ P. León, Traianeum de Italica, Sevilla 1988, p. 51; I. Rodá, «Los mármoles de Itálica...», cit., pp. 172-173.

${ }^{39}$ Cf. M. Mayer-I. Rodá, «The Use of Marble...», cit.

${ }^{40}$ Debemos agradecer al prof. J.M. Blázquez el envío de muestras del yacimiento de Castulo entre las cuales hay crustae de mármol de Almadén de la Plata, depositadas en el LEMLA de la UAB (núms. 629 y 637). 
Almadén de la Plata es el que se extrae en Macael, cerca de Olula del Río (Almería), en la sierra de Filabres ${ }^{41}$. Este último mármol tiene variedades listadas («cipollino») que se ha propuesto que hubieran sido explotadas en la Antigüedad, aunque las piezas de Italica a las que se atribuye este origen corresponden en realidad al «cipollino» de Caristo en la isla de Eubea ${ }^{42}$ y tampoco resulta del todo evidente la utilización de las variedades grises de este mármol ${ }^{43}$.

La utilización, en cambio, de la variedad blanca tiene una abundante documentación incluso analítica ${ }^{44}$. Por nuestra parte hemos observado la posible presencia de este material en un capitel del Museo de Almería y en una ménsula de Itálica ${ }^{45}$; no resulta clara su presencia en Munigua, Castulo y Cordu$b a$, y es en extremo sospechosa su identificación en el foro emeritense al lado de la utilización segura de mármol de Estremoz. ¿Qué sentido tendría para Mérida la muy dificultosa importación de mármol de Macael para un mismo programa iconográfico cuando disponía de las excelentes y próximas canteras del Alentejo?

Hemos de pensar que la semejanza del mármol de Macael con el de Almadén de la Plata y con las calidades blancas de Estremoz posibilita una fácil confusión y que éstos fueron objeto de una explotación oficial y mucho más generalizada.

A pesar de todo ello hay que reconocer que se da una dispersión del mármol de Macael por la vertiente sur de la sierra de Filabres. F. Braemer menciona la presencia de este mármol en la villa de Els Munts (Altafulla, Tarragona) y una estatua

${ }^{41}$ M. Cisneros, Mármoles hispanos..., cit., pp. 88-93; M.P. Lapuente-M. Cisneros-M. Ortiga, «Contribución a la identificación...», cit., pp. 257-274, para la identificación de diversas zonas de explotación; S.F. Ramallo-R. Arana, Canteras romanas de Carthago Nova.., cit., pp. 59, 64, mapa p. 66 fig. 7 para la dispersión en la zona de Murcia, y pp. 69 70 (inscripción de Cartagena).

42 El origen almeriense del mármol citado en el ara italicence de Cocceius fue propuesto por A.M. Canto, «Avances...», cit., p. 178. M. Cisneros (Mármoles hispanos..., cit., pp. 92-93) no se pronuncia al respecto. Por nuestra parte creemos que con toda seguridad el mármol caristio de las columnas mencionadas en este altar procede de la isla de Eubea; cf. I. Rodá, «Los materiales de construcción...», cit. p. 328, e Id., «Los mármoles de Itálica...», cit., pp. 157-158.

${ }^{43}$ En Munigua y en el teatro de Cádiz aparece un mármol gris que no parece coincidir con las muestras de que disponemos.

${ }^{4}$ P. Pensabene, «Costruzione pubbliche...», cit., p. 143 identifica como de mármol de Macael columnas del teatro de Málaga; M. Cisneros, Mármoles hispanos..., cit, pp. 143 147; M.P. Lapuente-M. Cisneros-M. Ortiga, «Contribución a la identificación...», pp. 257-274; S.F. Ramallo-R. Arana, Canteras romanas de Carthago Nova..., cit., pp. 58-59, fig. 3 .

${ }^{45}$ I. Rodá, «Los mármoles de Itálica...», cit., p. 158. del Museu Arqueològic de Catalunya en Barcelona fue considerada también como de este mármol, aunque en nuestra opinión se trata de mármol de Tasos ${ }^{46}$

Sin poner en entredicho la explotación y exportación romana del mármol de Macael blanco, aconsejaríamos prudencia al respecto y nos atreveríamos a limitar mucho su difusión dentro de la cuenca del Guadalquivir.

Como elemento de contraste, hemos recogido también un mármol de difusión mucho más limitada: el de Cabezo Gordo en la provincia de Murcia, el mármol por excelencia de Cartagena (Carthago Nova). Este mármol ha sido objeto de una buena monografía reciente y es fácilmente identificable macroscópicamente en el área limitada de uso, fechándose el inicio de su explotación en el siglo I a.C. ${ }^{47}$.

Los mármoles de la sierra de Mijas, en Mijas y Coín principalmente, han sido también objeto de un buen estudio con identificación de su área de dispersión ${ }^{48}$. Ambas variedades blancas de grano muy grueso son fácilmente distinguibles de cuantas hemos reseñado hasta ahora y lo son fácilmente entre sí dado que el de Coín adquiere una tonalidad más grisácea y su granulación es de tamaño superior.

En el mármol de Mijas se talló incluso escultura y en los Museos de Málaga y de Córdoba hay ejemplos de ello. Se halla presente también en Italica, en Hispalis (Sevilla), en la villa de Almedinilla (Córdoba) y en Almería en cuyo Museo hemos identificado un capitel. Cuatro esculturas del Museo de Málaga han sido identificadas como talladas en mármol de Coín ${ }^{49}$.

${ }^{46}$ F. Braemer, «L'ornementation...», cit., p. 70 y n. 43 para Tarragona y p. 62 y n. 85 , p. 72 para Els Munts; cf. Id. «Répertoire...», cit., p. 304 para la presencia de mármol de Macael en la villa de Els Munts que no consideramos en modo alguno probado. Para la estatua del Museo de Barcelona, cf. M. Mayer-A. Álvarez, «Le marbre grec comme indice pour les pièces sculptoriques grecques ou de tradition grecque en Espagne», Praktika. XII Congrès International d'Archéologie Classique (Atenas 1983), t. I, Atenas 1985, p. 184-190, esp. p. 189, muestra 580 del LEMLA, y M. Mayer, «Aproximación al problema...» cit., p. 273.

${ }_{47}$ S.F. Ramallo-R. Arana, Canteras romanas de Carthago Nova.., cit., pp. 52-59, pp. 62-63 para su uso y cronología, y pp. 68-69 para un inventario de los restos arqueológicos que usan como materia prima los mármoles del Cabezo Gordo y su área de dispersión.

${ }_{48}$ M.L. Loza-J. Beltrán, La explotación del mármol blanco..., cit., pp. 24-30 y mapa III.

${ }_{49}$ M.L. Loza-J. Beltrán, La explotación del mármol blanco..., cit, apéndice por M. Cisneros y M. Ortiga, pp. 78-79; D. Vaquerizo-J.M. Noguera, La Villa de El Ruedo, Almedinilla (Córdoba). Decoración escultórica e interpretación, Murcia 1997, núms. 2-5, pp. 112-129, núm. 10, pp. 144-145, núms. 17-24, pp. 174-193. 
Tal como exponemos en la parte analítica de este trabajo, los resultados que pueden obtenerse mediante el estudio isotópico permiten discriminar muy bien las muestras de Macael de las de Almadén de la Plata, lo cual viene a resolver el problema de su frecuente confusión macroscópica, aunque resulten también claramente diferenciadas mediante el análisis microscópico de láminas delgadas con luz polarizada ${ }^{50}$.

Las muestras de Cabezo Gordo no se distinguen en el análisis isotópico de las de Macael y de las de Almadén de la Plata. Su distinción petrológica es muy clara, sin embargo, y macroscópicamente incluso se puede alcanzar un alto grado de discriminación.

Las muestras correspondientes al anticlinal alentejano están claramente separadas en el análisis isotópico de las demás variedades, lo cual resulta ser un factor muy positivo, dada su confusión macroscópica con variedades de Almadén de la Plata, aunque su distinción en un análisis petrológico ya era evidente ${ }^{51}$.

Las peculiaridades de los mármoles de la Sierra de Mijas ya han sido destacadas y resultaría ocioso volver sobre ello. Los criterios puramente geológicos empleados no hacen en este caso distinción entre canteras.

El estudio que ahora presentamos dista mucho todavía de tener un carácter definitivo, aunque sí pretende delimitar la cuestión y recoger el estado actual de nuestros conocimientos sobre los materiales aquí tratados y los resultados de una técnica analítica actual que ya es mucho más que prometedora.

\section{CONCLUSIONES}

Nuestro estudio ha presentado las condiciones de dispersión y documentación de los materiales estu-

50 El método petrológico se verá, además, en el fututo objetivado con el desarrollo de la comparación mediante procesamiento digital de imagen; cf. para este método, A. Álvarez, «Clasificación automatizada de mármoles mediante procesamiento digital de imagen (Image Processing: Automatic Classification of Marbles)», Les marbres blancs..., cit., pp. 71-85 con bibliografía anterior de referencia; A. Álvarez- B. Obelić-A. Puig- D. Haye, «Determination of Provenance of Marbles used in Mediterranean Area», PACT 45 (1994) (pub. 1997), pp. 489-497; A. Álvarez-B. Obelić-D. Haye-J. Serrat, "Automatic Classification of Marbles by means of Digital Image Processing», Marmi Antichi II, cit., pp. 37-43.

${ }_{51}$ Para una descripción petrológica, cf. la de A. Álvarez en M. Mayer-I. Rodá, «The Use of Marble...». diados. Asimismo hemos reflejado en las notas el estado actual de los conocimientos sobre la explotación de estos mármoles en época romana. La andadura sobre el tratamiento de las canteras que hemos presentado no ha hecho más que comenzar y los datos que hoy exponemos se van a ampliar en el futuro.

En un breve plazo de tiempo deberíamos poder completar cuadros de circulación y dispersión de los materiales. El estudio de los restos arqueológicos de, y en, canteras se halla en un estadio incipiente y apenas conocemos algo de las posibles formas de explotación romanas. Habrá que fijar también criterios cronológicos para todos estos procesos.

Desde el punto de vista analítico se ha hecho evidente que la utilidad y fiabilidad del procedimiento empleado resultan ser limitadas. Como todos los métodos de análisis hasta ahora aplicados, el método isotópico proporciona unos datos significativos pero parciales, sobre todo cuando se superponen a los datos obtenidos en otras áreas geográficas del Imperio con cuyas variedades tienden a superponerse parcial o casi totalmente algunos mármoles hispanos. De todas maneras, como elemento discriminante entre variedades hispanas muy semejantes macroscópicamente, como el mármol de Macael, el de Estremoz y el de Almadén de la Plata, el sistema se revela relativamente eficaz; otra cuestión es el haberlos identificado previamente como materiales locales respecto a las variedades importadas, para lo cual es evidente que hay que recurrir a la petrología y a la experiencia arqueológica.

Sólo un cruce entre los datos obtenidos por distintos métodos de análisis puede proporcionar un criterio discriminatorio con alto grado de certeza; el análisis petrológico resulta dirimente en casos en que no lo es el análisis isotópico y este último presenta buenas posibilidades de separación entre materiales que ofrecen dudas en el microscopio. La experiencia arqueológica y en especial la familiaridad con los mármoles y sus canteras, así como sus vías de difusión, resulta indispensable como guía para orientar las líneas de búsqueda de los análisis.

A pesar de las limitaciones expuestas, a las que se suman las propias de nuestro estudio, no nos cabe duda de que esta aproximación promoverá el conocimiento de los mármoles tratados y esperemos que estimule nuevas aportaciones al tema y el estudio monográfico de las principales canteras que están a la espera de un tratamiento pormenorizado. 